\title{
Introduction of the hybcell-based compact sequencing technology and comparison to state-of- the-art methodologies for KRAS mutation detection
}

\author{
Agnes Zopf ${ }^{1, \dagger}$, Roman Raim ${ }^{2, \dagger}$, Martin Danzer ${ }^{1}$, Norbert Niklas', Rita Spilka ${ }^{3}$, Johannes Pröll1, \\ Christian Gabriel ${ }^{1}$, Andreas Nechansky², and Markus Roucka ${ }^{2}$ \\ ${ }^{1}$ Red Cross Transfusion Service for Upper Austria, Linz, Austria, ${ }^{2}$ VelaLabs, Vienna, Austria, and \\ ${ }^{3}$ Laboratory of Pathology Dr. Obrist \& Dr. Brunnhuber OG, Zams, Austria
}

${ }^{\dagger}$ A.Z. and R.R. contributed equally to this work

BioTechniques 58:126-134 (March 2015) doi 10.2144/000114264

Keywords: KRAS; compact sequencing; Hyborg; hybcell technology; next-generation sequencing

Supplementary material for this article is available at www.BioTechniques.com/article/114264.

The detection of KRAS mutations in codons 12 and 13 is critical for anti-EGFR therapy strategies; however, only those methodologies with high sensitivity, specificity, and accuracy as well as the best cost and turnaround balance are suitable for routine daily testing. Here we compared the performance of compact sequencing using the novel hybcell technology with 454 next-generation sequencing (454-NGS), Sanger sequencing, and pyrosequencing, using an evaluation panel of 35 specimens. A total of 32 mutations and 10 wild-type cases were reported using 454-NGS as the reference method. Specificity ranged from 100\% for Sanger sequencing to $80 \%$ for pyrosequencing. Sanger sequencing and hybcell-based compact sequencing achieved a sensitivity of $96 \%$, whereas pyrosequencing had a sensitivity of $88 \%$. Accuracy was $97 \%$ for Sanger sequencing, 85\% for pyrosequencing, and 94\% for hybcell-based compact sequencing. Quantitative results were obtained for 454-NGS and hybcell-based compact sequencing data, resulting in a significant correlation $(r=0.914)$. Whereas pyrosequencing and Sanger sequencing were not able to detect multiple mutated cell clones within one tumor specimen, 454-NGS and the hybcell-based compact sequencing detected multiple mutations in two specimens. Our comparison shows that the hybcell-based compact sequencing is a valuable alternative to state-of-the-art methodologies used for detection of clinically relevant point mutations.

In recent years, it has become evident that up-regulation or constant activation of the epidermal growth factor receptor (EGFR) contributes to tumor formation. For this reason, the EGFR has been targeted with a variety of therapeutics, such as gefitinib (Iressa), erlotinib (Tarceva), cetuximab (Erbitux), and panitumumab (Vectibix), with great success $(1,2)$. Nevertheless, in a significant number of patients, these drugs have little or no effect (3). The unresponsiveness of these patients might be due to mutations in signaling molecules downstream of the EGFR. A prominent example is the Kirsten rat sarcoma viral oncogene homolog (KRAS) gene, which shows a high mutational frequency in the hotspot regions of codons 12 and 13 (4-6). Moreover, codons 61, 146, and 154 have also been shown to play an important role in certain cancer types (6). Mutations in codons 12 and 13 result in a conforma- tional change in the KRAS protein. As a consequence, its dephosphorylation is inhibited, leading to constitutive activation and thus making therapeutic interventions targeting its upstream regulator, EGFR, ineffective (6).

For that reason, and to ensure effective therapeutic intervention, molecular biological methods for detecting variations in codons 12 and 13 of the KRAS gene are now used (7-13). Solid tumors

\section{METHOD SUMMARY}

In the present study, we evaluated hybcell-based compact sequencing for KRAS mutation analysis and compared this approach with Sanger sequencing, pyrosequencing, and next-generation sequencing. Our data show that hybcell-based compact sequencing is a cost-effective microarray-based method for detecting clinically relevant KRAS point mutations in tumor material. 
are very heterogeneous, containing tumor cells, nonneoplastic cells, inflammatory cells, vascular endothelial cells, and mesenchymal cells. Therefore, a sensitive and reliable method is needed to detect somatic variants at low frequencies in a wild-type background (14). Additionally, to be widely used and accepted, the best-in-class methodologies have to be competitive in terms of assay costs and turnaround time in addition to having high sensitivity and accuracy (15).

To address some or all of these requirements, many commercial as well as in-house tests have been developed. These approaches include conventional Sanger sequencing, pyrosequencing, next-generation sequencing (NGS), and PCR-based methods. Each method meets some but not all of the aforementioned requirements. To meet all of these criteria, a novel technology called hybcell, which uses the Hyborg instrument (Anagnostics Bioanalysis GmbH, St. Valentin, Austria), was invented.

The aim of this study was to determine which of the following four technologies is best suited for routine diagnostics by testing each using the same tumor material: compact sequencing using the novel hybcell technology, 454 next-generation sequencing (454-NGS), the wellestablished Sanger sequencing method, or the PyroMark system.

\section{Materials and methods}

\section{Biopsies}

An evaluation panel of 35 formalin-fixed paraffin-embedded sections $(2 \times 5 \mu \mathrm{m})$ was provided from the tissue archive of the pathology laboratory of Obrist and Brunhuber OG (Zams, Tyrol). No patient data were included. The only reported feature common to all of the provided samples was a minimum tumor content of $20 \%$ in the biopsy material (Table 1). For 7 of the 35 specimens, the tumor content was not specified.

\section{DNA isolation}

DNA was extracted from the sections using the QIAamp DNA FFPE tissue kit (Qiagen, Hilden, Germany) according to the manufacturer's manual. DNA isolates were eluted in $70 \mu \mathrm{L}$ elution buffer. DNA content was determined using a NanoDrop 2000 spectrophotometer (Thermo Scientific, Waltham, MA) or the BioPhotometer plus (Eppendorf, Hamburg, Germany). The amount of DNA used in the hybcell assays ranged from 99-2340 ng. However, DNA concentration is only of partial importance, as the assay has internal quality control checks to determine if the total DNA content after PCR amplification is sufficient to provide reliable data. All of the presented results had a valid assay control, indicating that the total amount of DNA applied was sufficient for reliable data evaluation.

Hybcell-based compact sequencing Compact sequencing using the microarray-based hybcell system allows the detection of seven clinically relevant $K R A S$ mutations: p.G12A, p.G12D, p.G12R, p.G12C, p.G12S, p.G12V, and p.G13D. The assay is split into two parts: (i) PCR amplification and labeling of the target sequence from the template DNA (duplex PCR) and (ii) hybridization of single-stranded PCR products to short oligonucleotides (primers) printed onto a cylindrical plastic carrier (core). These primers are specific for the KRAS mutations indicated above and are attached to the cylinder at their $5^{\prime}$ ends. The core is divided into two halfcylinders, which are separated by baffles used for efficient mixing of the sample solution.

During processing, the core is inserted into a sample reservoir (tube). This core-tube assembly is then introduced into the analysis chamber, which is covered by two heating bars allowing temperature adjustment. The primers hybridize to the $3^{\prime}$ ends of the labeled single-stranded PCR products; if a primer is bound to its cognate PCR product, it is then extended by DNA polymerase, resulting in tighter binding to the PCR product. During analysis, the sample is passed over the printed surface by rotation of the core within the tube. After hybridization and primer extension, weakly bound and unbound PCR products are washed away in a stringent washing step. The fluorescence signal (excitation wavelength: $635 \mathrm{~nm}$ ) of the labeled, hybridized PCR strands is then measured using a CCD camera (Figure 1). Scanning of the entire core is achieved by rotation and taking individual pictures of the different surface areas. These single pictures are subsequently arranged into a full two-dimensional representation of the core surface.
For amplification and labeling of DNA, the hybcell Oncogenes Tissue plex A-1-02 kit (Anagnostics Bioanalysis $\mathrm{GmbH}$ ) was applied using an MJ Mini Thermal Cycler (Bio-Rad Laboratories, Hercules, CA). For the PCR reaction, $12.5 \mu \mathrm{L}$ ready-touse PCR-mix (including DNA polymerase, nucleotides, and primers) was mixed with 7 $\mu \mathrm{L}$ template DNA and $5.5 \mu \mathrm{L}$ DNA dilution buffer. Because the subsequent hybridization reaction requires single-stranded DNA, an asymmetric PCR approach was applied. The PCR protocol was performed in accordance with the manufacturer's protocol. The amplification and labeling protocol started with a denaturation step at $94^{\circ} \mathrm{C}$ for $120 \mathrm{~s}$, followed by 38 cycles of $94^{\circ} \mathrm{C}$ for $20 \mathrm{~s}, 61^{\circ} \mathrm{C}$ for $20 \mathrm{~s}$, and $72^{\circ} \mathrm{C}$ for $60 \mathrm{~s}$. The PCR primers were labeled with Cy5, allowing detection of the PCR product after hybridization and washing. After amplification, the PCR product was mixed with primer extension mix (containing nucleotides and polymerase). This reaction mixture was then passed over the hybcell surface. Automated processing was performed using the Hyborg instrument (Anagnostics Bioanalysis $\mathrm{GmbH}$ ). For the first 2.5 min of processing, the temperature of the heating bars was set to $108^{\circ} \mathrm{C}$ for the denaturation of double-stranded DNA and the activation of polymerase. A 390 $\mu \mathrm{L}$ volume of primer extension (PE) buffer (7 $\mathrm{mM} \mathrm{MgCl}_{2}$ ) was introduced into the tube via the tubing system. The temperature was then set to $63^{\circ} \mathrm{C}$ (hybridization + primer extension) for $23 \mathrm{~min}$. At the beginning of this incubation period, an additional $40 \mu \mathrm{L}$ PE buffer was added. After this incubation, $6.5 \mathrm{~mL}$ wash buffer was added to the tube (liquid addition and aspiration at the same time to maintain tube volume), resulting in the removal of unbound DNA. A short temperature increase $(10 \mathrm{~s})$ to $84^{\circ} \mathrm{C}$ and an additional $6.5 \mathrm{~mL}$ wash buffer resulted in removal of weakly bound DNA (not primer extended). The temperature was decreased to $30^{\circ} \mathrm{C}$ for $5 \mathrm{~s}$, and $2 \mathrm{~mL}$ wash buffer was pumped into the tube before scanning of the core surface with an exposure time of $400 \mathrm{~ms}$. The data generated were analyzed with the hybcell KRAS DNA plexA CE-IVD software (Anagnostics Bioanalysis GmbH).

\section{PyroMark sequencing}

Using the gene-specific primers KRAS forward [5'-AGAGGCCTGCTGAAAATGACTGAA-3'] and KRAS 
Table 1. Summary of results obtained from four different methods used for KRAS mutation analysis of 35 tumor samples.

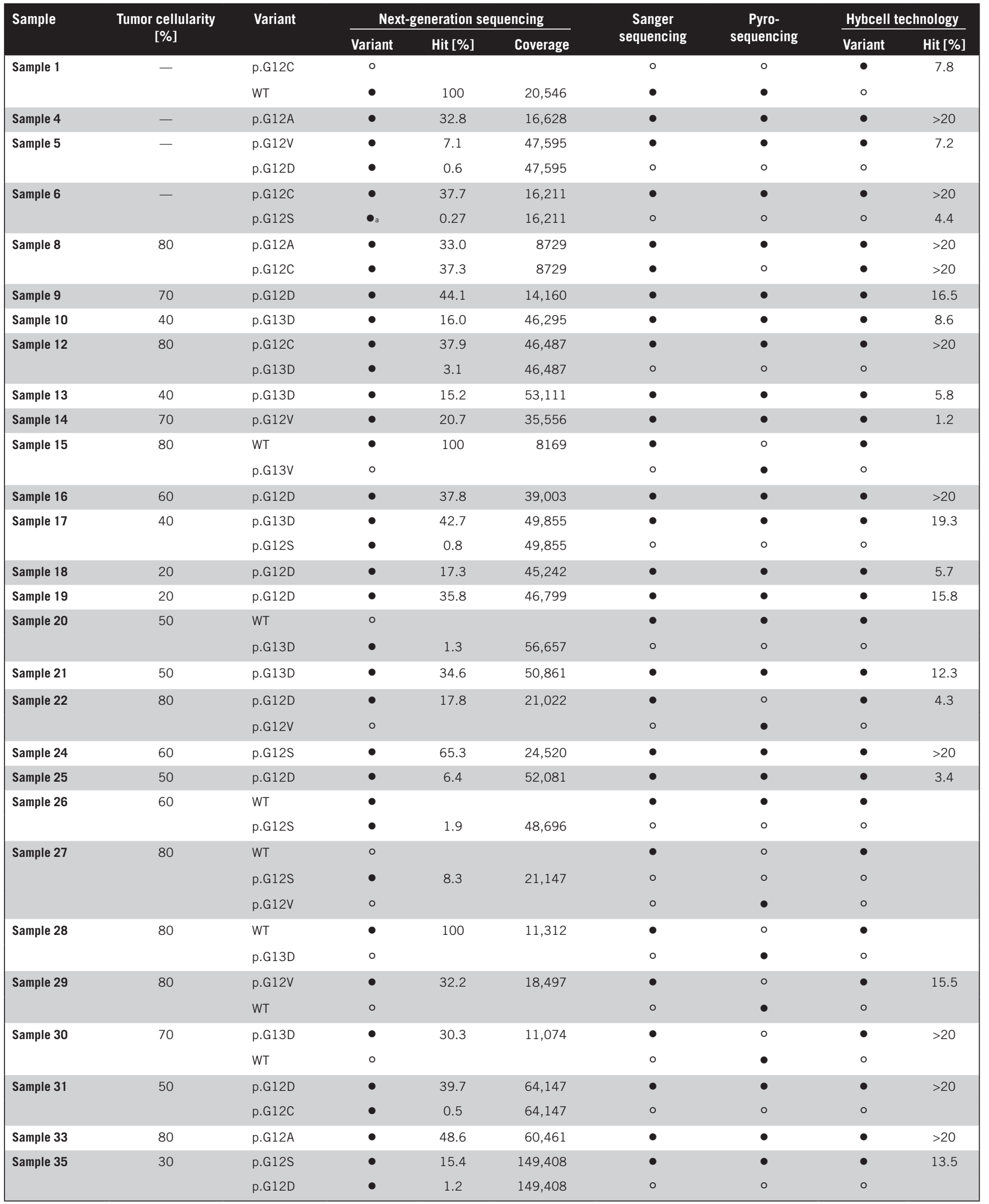

Some of the samples analyzed show contradictory results throughout the different assays, indicating differences in method performance. All variants investigated with next-generation sequencing (NGS) with a frequency $>5 \%$ were included in statistical analysis. Samples $2,3,7,11,23,32$, and 34 showed no aberrations for all methods in codons 12 and 13 of the KRAS gene; those data are not displayed in the table. The coverage of those wild-type samples ranged from 46,682 to 72,532 reads (mean coverage: 56,092 reads) per sample. ${ }^{\bullet}$ : variation was detected; ${ }^{\circ}$ : variation was not detected.

${ }^{a}$ Frequency below threshold of $0.5 \%$; WT: wild-type; coverage: total read count per sample. 


\section{BLOW UP THE HOLDUPS} IN YOUR RNA RESEARCH

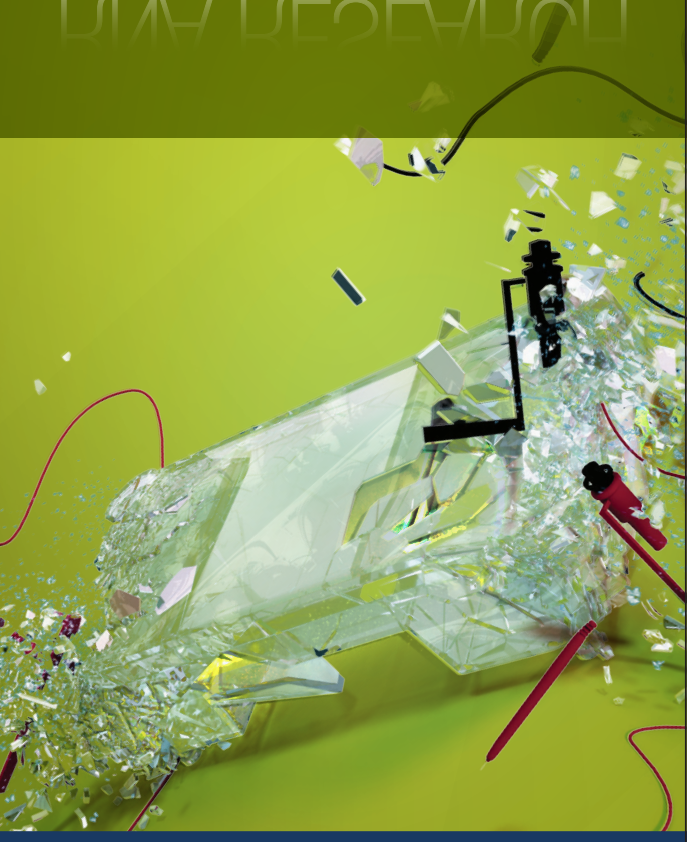

Assess RNA quality and quantity simultaneously.

\section{FULLY AUTOMATED}

\section{FRAGMENT ANALYZER ${ }^{\text {TM }}$} DOES IT ALL.

\section{Quantifies with precision (10\% CV)} and accuracy $( \pm 20 \%)$

Provides an RNA quality number that correlates to legacy data

Observes gDNA contamination in RNA

Calculates the Illumina $\mathrm{DV}_{200}$ metric for FFPE samples

No chips. No tapes. No compromises.

More at AATI-US.COM

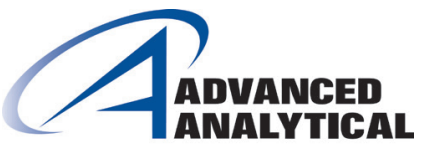

A
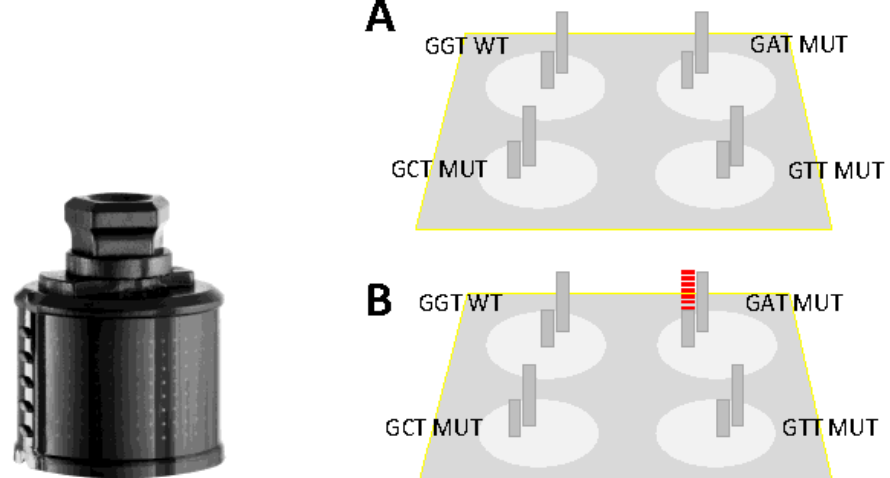

Hybridization

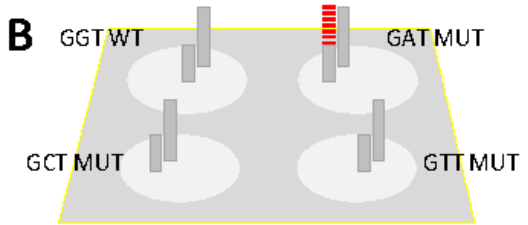

Primer Extension

Hybcell

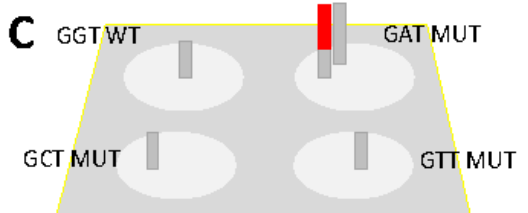

Stringent Wash

Figure 1. Schematic diagram of the hybcell-based compact sequencing technology. DNA is amplified and labeled using a common thermocycler followed by the introduction of the generated amplicons into the hybcell. (A) Labeled amplicons hybridize to the primers spotted on the surface of the hybcell. (B) Only those DNA molecules with a perfect match in KRAS codons 12 and 13 are extended. (C) The others are removed after a stringent wash, and the signal of the labeled and bound mutated DNA molecules is then measured.

reverse biotin [5'-TTAGCTGTATCGTCAAGGCACTCT-3'], PCR was performed on 5-10 $\mathrm{ng} / \mathrm{\mu L}$ template DNA with a Type-it HRM Mastermix (Qiagen). The cycling profile comprised an activation step at $95^{\circ} \mathrm{C}$ for $5 \mathrm{~min}$, followed by 40 cycles of amplification, including $30 \mathrm{~s}$ at $95^{\circ} \mathrm{C}, 20 \mathrm{~s}$ at $58^{\circ} \mathrm{C}$, and $30 \mathrm{~s}$ at $72^{\circ} \mathrm{C}$ on a Rotor-Gene $Q$ cycler (Qiagen). A melt analysis was performed after the PCR as a quality control. PCR products were purified via Sepharose beads on the PyroMark Q24 Working Station (Qiagen) and sequenced in the PyroMark Q24 using the sequencing primer KRAS seq forward [5'-TGTGGTAGTTGGAGCT-3']. The default sequence to analyze was set to [5'-G/NGTGGCGTAG-3'], with the dispensation order of [5'-ACTGACTACGATCGTA-3'] enabling a later transversion to [5'-GG/NTGGCGTAG-3'], [5'-GGTG/NGCGTAG-3'], or [5'-GGTGG/ NCGTAG-3'] to analyze every common mutation occurring in codons 12 and 13 of the KRAS gene.

Target amplification and purification for Sanger sequencing and 454-NGS For amplification of KRAS codons 12 and 13 , a reaction mix containing $0.5 \mu \mathrm{M}$ forward primer [5'-CGTATCGCCTCCCTCGCGCCATCAG-MIDAGGCCTGCTGAAAAGACTG-3'], 0.5 $\mu \mathrm{M}$ reverse primer [5'-CTATGCGCCTT-
GCCAGCCCGCTCAG-MID-GTTTCTTACCAGGACGTGGTC-3'], $2 \mu \mathrm{L}$ 10× buffer including $18 \mathrm{mM} \mathrm{MgCl}^{2}, 0.2 \mathrm{mM}$ of each dNTP, 5\% DMSO, $1 \mathrm{U} / \mu \mathrm{L}$ FastStart High Fidelity polymerase (FastStart High Fidelity PCR System, Roche Applied Science, Penzberg, Germany), and 50 ng DNA template in a total volume of $25 \mu \mathrm{L}$ were run on a Verity thermal cycler (Life Technologies, Carlsbad, CA) with the following protocol: preheating to $94^{\circ} \mathrm{C}$, followed by 40 cycles of $94^{\circ} \mathrm{C}$ for $15 \mathrm{~s}, 55^{\circ} \mathrm{C}$ for $15 \mathrm{~s}, 72^{\circ} \mathrm{C}$ for $30 \mathrm{~s}$, and a final extension at $72^{\circ} \mathrm{C}$ for $7 \mathrm{~min}$ followed by cooling to $4^{\circ} \mathrm{C}$. PCR products were purified using Agencourt AMPure XP beads (Beckman Coulter, Vienna, Austria) according to the Amplicon Library Preparation Method Manual, GS FLX Titanium Series (October 2009; 454 Life Sciences, Roche, Brandford, CT). Purified amplicons were used for further Sanger sequencing as well as 454-NGS.

\section{Sanger sequencing}

Sanger sequencing was performed using the ABI PRISM 3130xL Genetic Analyzer (Life Technologies). Direct cycle sequencing was performed on a Verity Thermocycler (Life Technologies) in a 10- $\mu \mathrm{L}$ reaction volume containing 2 $\mu \mathrm{L}$ purified PCR products, $1 \mu \mathrm{L}$ BigDye Terminator v1.1 chemistry (Life Technologies), $1 \times$ sequencing buffer, and $2 \mu \mathrm{M}$ 
of the respective sequencing primers: forward primer [5'-CGTATCGCCTCCCTCGCGCCATCAG-3'] and reverse primer [5'CTATGCGCCTTGCCAGCCC GCTCAG-3']. Prior to Sanger sequencing, all reactions were purified using Sephadex G-50 (Sigma-Aldrich, St. Louis, MO). Finally, the data were analyzed using Lasergene SeqMan Software (DNASTAR Inc., Madison, WI).

\section{4-NGS}

454-NGS was performed using the GS FLX+ instrument (454 Life Sciences). AMPure XP bead-purified PCR products were quantified by Quant-iT PicoGreen dsDNA Reagent (Life Technologies) using an Infinite 200Pro fluorometer (Tecan, Grödig, Austria). The amplicon intensity signals were translated from nanograms per microliter (ng/ $\mu \mathrm{L}$ ) to molecules per microliter (molecules $/ \mu \mathrm{L}$ ), and two multiplex identifier (MID) tagged samples were pooled in equimolar amounts and diluted to a final concentration of $1 \times 10^{6}$ molecules/ $\mu \mathrm{L}$. Emulsion PCR (emPCR), bead recovery, and bead enrichment were performed according to the emPCR Amplification Method Manual-Lib-A SV (January 2010) and Technical Bulletin TCB No. 2011-001 (454 Life Sciences, Roche, Brandford, CT) and carried out on a GeneAmp PCR System 9700 (Life Technologies). Afterward, bead recovery was performed manually, whereas bead enrichment was done with the robotic enrichment module (454 Life Sciences) on a Microlab STARlet robot (Hamilton Robotics GmbH, Martinsried, Germany). After automated enrichment, bead counting was performed using the Scepter 2.0 (Merck Millipore, Billerica, MA), resulting in the loading of 340,000 beads in 1 of 8 regions of the Pico Titer Plate (454 Life Sciences). 454-NGS was carried out according to the appropriate sequencing method manual (Sequencing Method Manual XLR70, August 2012; 454 Life Sciences) on the GS FLX+ instrument (454 Life Sciences). The sequencing data were aligned to the KRAS reference sequence (NC_000012.11 obtained from NCBI) and analyzed using the GS Amplicon Variant Analyzer version 2.9 (454 Life Sciences). All variants with $\geq 0.5 \%$ hit percentage were examined. Only mutations displayed in both sequencing directions were included for variant calling.
Statistical analysis

Specificity, sensitivity, accuracy, and precision were calculated with a $2 \times 2$ contingency table using 454-NGS as the reference method. The Pearson correlation was used to compare qualitative test results from all four methods, and the available quantitative results generated by 454-NGS and hybcell-based compact sequencing as well as the percentage of tumor cells were tested for significance of correlation.

\section{Results and discussion}

The clonal heterogeneity of solid tumors can be difficult to determine (16-18). Identification of a small subset of mutated cells $(<10 \%)$ as well as multiple mutated cell clones in a single tumor sample is challenging for many molecular biological methods (3,6,19-21). The false-negative detection of mutated cells may result in treatment resistance or the use of inappropriate anti-EGFR antibodies for treatment (16-18). Recently, Chen et al. showed that patients carrying an amino acid change in codon 13 of the KRAS gene are more sensitive to anti-EGFR treatment than patients with codon 12 mutations (22). Based on this treatment-relevant fact, methods detecting low frequency and multiple mutations have to be implemented in routine analysis. In general, patients showing low KRAS-mutated cell clone populations should not be excluded from anti-EGFR therapy. Anti-EGFR therapy may be used for triggering tumor shrinkage by targeting wild-type clones. This enhances the accessibility of co-administered chemotherapeutics targeting molecules downstream of the KRAS protein. Also, the sensitivity of KRAS-mutated tumor cells for radiotherapy may be increased.

An evaluation panel of 35 specimens was tested with each of the 4 detection methods, and the results are shown in Table 1. Results generated with 454-NGS and the novel hybcell-based compact sequencing technology are qualitative and quantitative (absolute and relative), whereas only quali- tative results were obtained from Sanger sequencing as well as pyrosequencing. 454-NGS, used as the reference method in this approach, identified 10 wild-type samples and 25 samples carrying point mutations in codons 12 and 13 of the KRAS gene. Single mutations were detected in 18 cases, whereas 7 samples carried 2 mutations, resulting in 2 different mutated cell clones. The 32 mutations encompassed the following types: p.G12A ( $n=$ 3), p.G12V $(n=3)$, p.G12D $(n=9)$, p.G12C $(n=5)$, p.G12S $(n=5)$, and p.G13D $(n=7)$.

For the comparison of 454-NGS with the other 3 methods, only variants with a frequency $>5 \%$ were included for further analysis, which was true for 24/32 mutations. Sanger sequencing confirmed the same 10/10 wild-type cases as 454-NGS, whereas pyrosequencing only identified 8/10 and the novel hybcell-based compact sequencing technology 9/10. The specificity of the different detection methods ranged from 100\% for Sanger sequencing to $80 \%$ for pyrosequencing (Table 2). Twenty-three of 24 mutated cases were detected by Sanger sequencing and the hybcell-based compact sequencing resulting in a sensitivity of $96 \%$. Compared with 454-NGS, pyrosequencing achieved a sensitivity of $88 \%$ (21/24 cases). The accuracy, which describes true results over total results, was $97 \%$ for Sanger sequencing, 85\% for pyrosequencing, and $94 \%$ for hybcell-based compact seque Introduction of the hybcell-based compact sequencing technology and comparison to state-of-the-art methodologies for KRAS mutation detection ncing. Moreover, the precision (true-positive over total positive results) ranged from 100\% to $91 \%$ (Table 2). A Pearson correlation of 0.971 indicated a high degree of agreement of Sanger sequencing with the reference method. An adequate correlation of 0.858 was achieved by hybcell-based compact sequencing, whereas the Pearson correlation for pyrosequencing was only 0.657 .

Several groups have previously carried out evaluations of multiple KRAS detection methods in similar contexts $(10,11,23)$.

Table 2. Specificity, sensitivity, accuracy, precision, and Pearson correlation of Sanger sequencing, pyrosequencing and hybcell-based compact sequencing measured using 454-NGS as reference method.

\begin{tabular}{|lccccc|} 
Method & Specificity & Sensitivity & Accuracy & Precision & Pearson correlation \\
\hline Sanger sequencing & $100 \%$ & $96 \%$ & $97 \%$ & $100 \%$ & 0.971 \\
$\begin{array}{l}\text { Pyrosequencing } \\
\begin{array}{l}\text { Hybcell-based compact } \\
\text { sequencing }\end{array}\end{array}$ & $80 \%$ & $88 \%$ & $85 \%$ & $91 \%$ & 0.657 \\
\hline
\end{tabular}




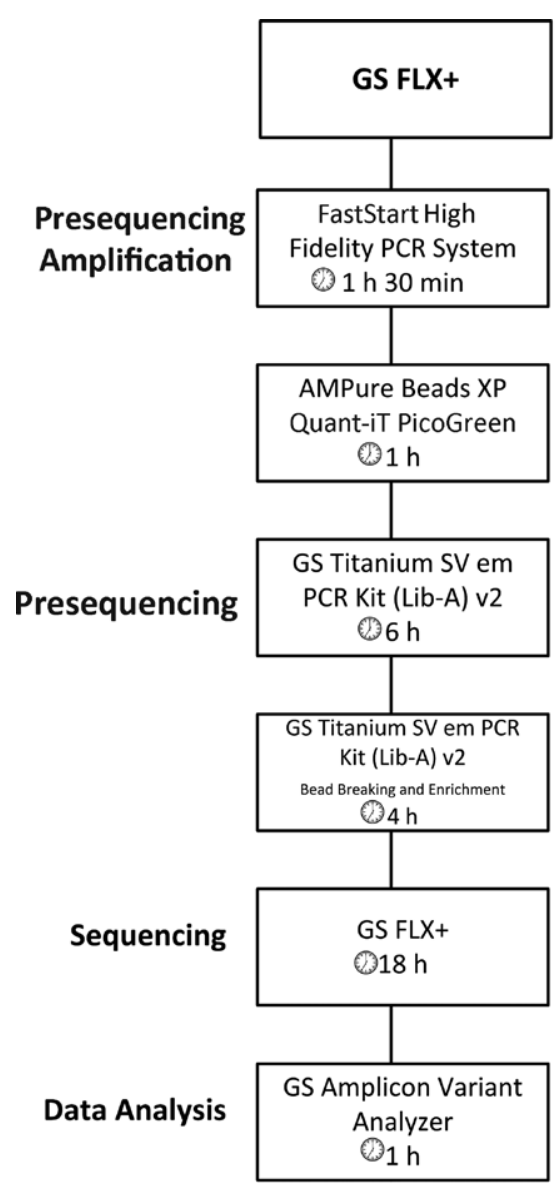

Hands-on time

Duration
$4 \mathrm{~h} 30 \mathrm{~min}$

$30 \mathrm{~h} 30 \mathrm{~min}$

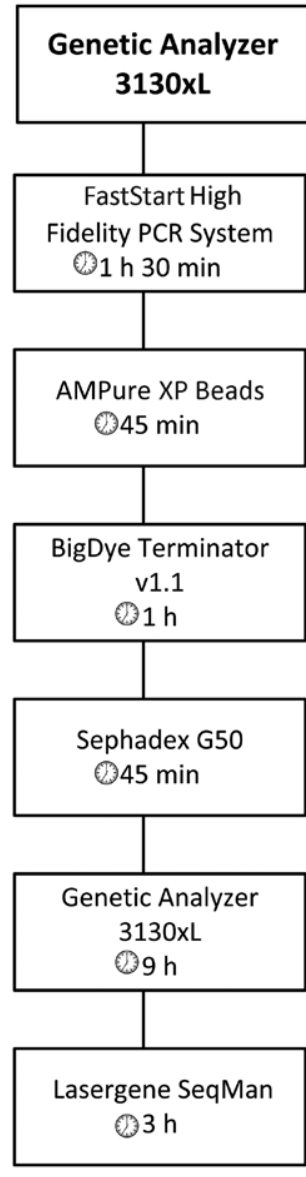

$2 \mathrm{~h}$

$15 \mathrm{~h}$

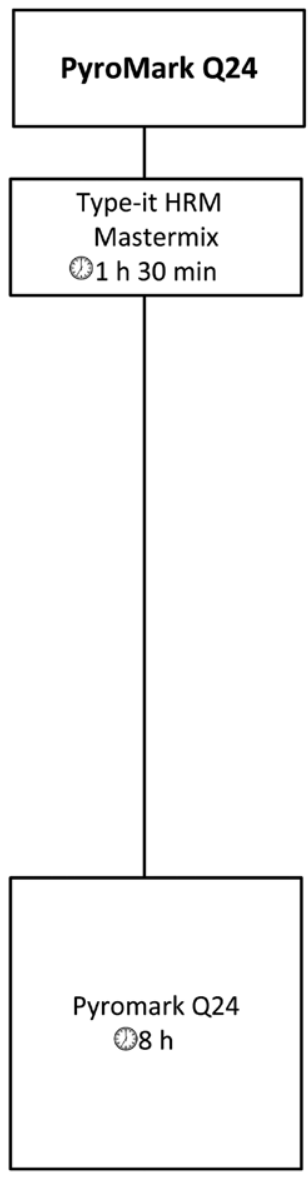

$1 \mathrm{~h}$

$9 \mathrm{~h} 30 \mathrm{~min}$

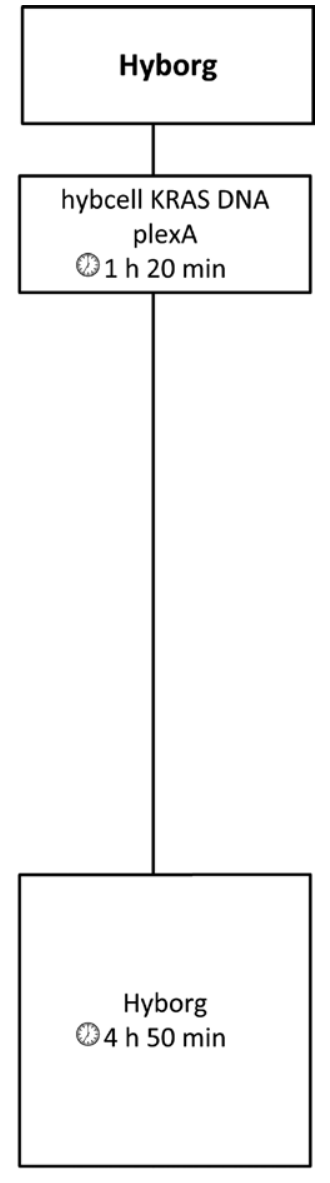

$45 \mathrm{~min}$

$6 \mathrm{~h}$

Figure 2. Overview of the workflow and turnaround time of 454-NGS (GS FLX+), Sanger sequencing (Genetic Analyzer 3130xL), pyrosequencing (PyroMark Q24), and hybcell-based compact sequencing.

Altimari et al. (23) identified Sanger sequencing as the least sensitive (85\%) and accurate (90\%) detection method, whereas Sanger sequencing showed the best results in our study (Table 2). In contrast, PyroMark Q24 sequencing showed the poorest performance in our study, while pyrosequencing demonstrated the best performance in the study by Altimari et al. (23). However, compact sequencing using hybcells showed good results in terms of sensitivity (96\%), specificity (90\%), accuracy (94\%), and precision $(96 \%)$ in the present study. The different results indicate that every KRAS testing facility has to evaluate the KRAS detection performance of each assay with the given sample material, instruments, and possible working regulations $(10,11)$.

The quantitative results define the percentage of mutated cell clone populations versus the wild-type population and were obtained with 454-NGS as well as with hybcell-based compact sequencing (Table 1). While 454-NGS classifies the results from $0 \%$ to $100 \%$, hybcell-based compact sequencing gives information about clone sizes from $5 \%$ to $20 \%$, whereas all clones over $20 \%$ are declared $\geq 20 \%$. Sanger sequencing and pyrosequencing are able to detect all variations in the amplified DNA sequences, but mutant copies must have a concentration of at least 20\% for Sanger sequencing and $5 \%$ for pyrosequencing $(10,14)$. The comparison of the qualitative results of 454-NGS and the hybcell-based compact sequencing technology reveals a significant correlation, $r=0.914$. All four methods detected the p.G12D amino acid change in sample 25. 454-NGS determined this variation in $6.4 \%$ of 52,081 reads. Sample 27 had a p.G12S mutation in $8.3 \%$ of 21,147 reads analyzed with 454-NGS, but Sanger sequencing and hybcell-based compact sequencing generated false-negative results, and pyrosequencing indicated an additional false-positive amino acid change (p.G12V). The percentage of tumor cells was reported for 28 of 35 cases. The comparison of the tumor cell content and the qualitative results of NGS $(r=0.023)$ and the hybcell-based compact sequencing $(r$ $=-0.14$ ) indicates no significant correlation. The percentage of tumor cells is the relative amount of tumor cells compared to other cells. This tumor cell content is assessed by microscopy of histologically prepared tissue samples from pathologists. Because they are only subjective estimates of tumor cell content, these percentages are often not accurate, as described by Smits et al. (24). To avoid these misinterpretations, the use of quantitative molecular biological techniques would improve the verification of the tumor cell content. 
Cell clones $<1 \%$ in frequency were indicated in samples 5, 6, 17, and 31 by 454-NGS. The mean of the absolute read counts carrying these mutations was 359 reads. The detection of those low-level mutations could not be achieved by any of the other three methods. In such cases, only 454-NGS, as presented in this study, or PCR-based methods such as real-time PCR can detect mutant alleles below 5\% $(13,25,26)$.

Moreover, the identification of multiple mutations in a tumor sample is very challenging (16-18). In our study multiple, mutations were observed in sample 6 (p.G12C, p.G12S) and sample 8 (p.G12A, p.G12C). 454-NGS and hybcell-based compact sequencing showed both variations in sample 8, with frequencies $>20 \%$. Because of their high frequency, both variations were clearly visible by Sanger sequencing but could not be detected with pyrosequencing. In sample 6, the p.G12S mutation was detected with hybcell-based compact sequencing at a frequency of $4.4 \%$, whereas $454-N G S$ displayed this variant in only $0.27 \%$ of 16,211 reads. Although this is below the frequency threshold (>0.5\%) used in our analysis, it was observed in both sequencing directions. Detection of multiple as well as low-level mutations within the same sample presents a problem for conventional Sanger sequencing (limit of detection $20 \%$ ), as peaks near the baseline are difficult to interpret. This ultimately might lead to falsepositive or false-negative results. Moreover, multiple mutations cannot be separated and assigned to different cell clones with Sanger sequencing. To elucidate the haplotypes of multiple variants, the results have to be reanalyzed with 454-NGS or hybcellbased compact sequencing.

Along with high sensitivity and specificity, only assays with the best cost and turnaround times are used for routine clinical testing. A comparative overview of the workflow of all four methods used in this study is given in Figure 2. All four technologies use DNA isolation as well as specific PCR amplification prior to mutation analysis. Whereas for 454-NGS or Sanger sequencing several presequencing steps such as purification, quantification, cycle sequencing (Sanger sequencing), or emPCR (454-NGS) have to be performed, amplicons generated for hybcell-based compact sequencing and pyrosequencing (PyroMark Q24) can be sequenced immediately after amplification, resulting in a faster workflow. 454-NGS using the high-throughput GS FLX+ instrument is labor-intensive and time-consuming, and large amounts of reagents are consumed, making it the most expensive technology among those compared. On the other hand, 454-NGS generates very sensitive results and has the ability to identify multiple mutant subclones in one tumor sample. Such detailed data, which at the moment only NGS can provide, enable personalized cancer treatment (27). However, the cost and turnaround time of the GS FLX+ is suitable for daily clinical routine testing only for labs with high-throughput or highly sophisticated infrastructure settings. For the routine testing of KRAS codons 12 and 13, other NGS instruments such as the GS Junior (454 Sciences), MiSeq (Illumina, San Diego, CA) or lonTorrent (Life Technologies) would be more suitable $(13,23,27,28)$. If high-throughput technology is not required, hybcell-cased compact sequencing, Sanger sequencing (Genetic Analyzer 3130xL), or pyrosequencing (Pyromark Q24) are alternatives. The average handson-time of the different technologies range from $45 \mathrm{~min}$ (Hyborg) to $4 \mathrm{~h} 30 \mathrm{~min}$ (GS FLX+). For daily routine testing, the hands-on-time as well the entire testing time is important to guarantee a reliable and rapid appraisal of clinical samples. With respect to data analysis, hybcellbased compact sequencing and pyrosequencing (Pyromark Q24) generate a final mutation status report on the sequenced samples and no further analysis steps are necessary. In contrast, 454-NGS and Sanger sequencing data have to be analyzed with additional software tools. The strength of compact sequencing is the generation of results within $6 \mathrm{~h}$ and the cost-effective analysis of single samples without reagent waste, ideal for facilities with a small to moderate sample throughput.

Based on our findings, we conclude that the hybcell-based compact sequencing technology represents a valuable alternative to the existing methods used for the detection of clinically relevant point mutations in the KRAS gene. The technique is characterized by its high sensitivity, its ability to independently detect multiple KRAS mutations within the same sample, as well as its easy and fast performance. While Sanger sequencing provides only a qualitative result, hybcell-based compact sequencing and NGS generate quantitative results. Moreover, the novel hybcell-based compact sequencing technology offers a cost-effective assay for single samples without reagent waste. Furthermore, the technology can be extended to additional mutations such as NRAS, BRAF, or PIK3CA, which are also known to be of predictive importance for cancer diagnosis. This extension may be accomplished by printing additional primers onto the same hybcell core but would require almost identical cycling conditions for the different mutations investigated. Therefore, it may be more appropriate to introduce separate test kits printing the mutations of interest on separate hybcell cores.

\section{Author contributions}

A.Z. and R.R. wrote the manuscript. A.Z. performed GS FLX+ and Sanger sequencing. R.R. performed compact sequencing and RS pyrosequencing. N.N. analyzed GS FLX+ data and performed statistical analysis. J.P. and M.D. designed the study and significantly contributed to the discussion. M.R., A.N., and C.G. critically reviewed the manuscript.

\section{Acknowledgments}

This work was funded by Austrian Research Promotion Agency (FFG) COIN project (KONMODI 827279).

\section{Competing interests}

The authors declare no competing interests.

\section{References}

1. Bogdan, S. and C. Klambt. 2001. Epidermal growth factor receptor signaling. Curr. Biol. 11:R292-R295.

2. Ciardiello, F. and G. Tortora. 2008. EGFR antagonists in cancer treatment. N. Engl. J. Med. 358:1160-1174.

3. Chang, D.Z., V. Kumar, Y. Ma, K. Li, and S. Kopetz. 2009. Individualized therapies in colorectal cancer: KRAS as a marker for response to EGFR-targeted therapy. J. Hematol. Oncol. 2:18.

4. Gschwind, A., O.M. Fischer, and A. Ullrich. 2004. The discovery of receptor tyrosine kinases: targets for cancer therapy. Nat. Rev. Cancer 4:361370.

5. McGrath, J.P., D.J. Capon, D.H. Smith, E.Y. Chen, P.H. Seeburg, D.V. Goeddel, and A.D. Levinson. 1983. Structure and organization of the human Ki-ras proto-oncogene and a related processed pseudogene. Nature 304:501-506.

6. Normanno, N., S. Tejpar, F. Morgillo, A. De Luca E. Van Cutsem, and F. Ciardiello. 2009. Implica- 
tions for KRAS status and EGFR-targeted therapies in metastatic CRC. Nat Rev Clin Oncol. 6:519-527.

7. Angulo, B., E. Garcia-Garcia, R. Martinez, A Suarez-Gauthier, E. Conde, M. Hidalgo, and F. Lopez-Rios. 2010. A commercial real-time PCR kit provides greater sensitivity than direct sequencing to detect KRAS mutations: a morphology-based approach in colorectal carcinoma. J. Mol. Diagn. 12:292-299.

8. Arcila, M., C. Lau, K. Nafa, and M. Ladanyi. 2011. Detection of KRAS and BRAF mutations in colorectal carcinoma: roles for high-sensitivity locked nucleic acid-PCR sequencing and broadspectrum mass spectrometry genotyping. J. Mol. Diagn. 13:64-73.

9. Tuononen, K., S. Maki-Nevala, V.K. Sarhadi, A. Wirtanen, M. Ronty, K. Salmenkivi, J.M. Andrews, A.I. Telaranta-Keerie, et al. 2013. Comparison of targeted next-generation sequencing (NGS) and real-time PCR in the detection of EGFR, KRAS, and BRAF mutations on formalin-fixed, paraffin-embedded tumor material of non-small cell lung carcinoma-superiority of NGS. Genes Chromosomes Cancer 52:503-511.

10. Franklin, W.A., J. Haney, M. Sugita, L. Bemis, A. Jimeno, and W.A. Messersmith. 2010. KRAS mutation: comparison of testing methods and tissue sampling techniques in colon cancer. J. Mol. Diagn. 12:43-50.

11. Gonzalez de Castro, D., B. Angulo, B. Gomez, D. Mair, R. Martinez, A. Suarez-Gauthier, F. Shieh, M. Velez, et al. 2012. A comparison of three methods for detecting KRAS mutations in formalin-fixed colorectal cancer specimens. Br. J. Cancer. 107:345-351.

12. Bihl, M.P., S. Hoeller, M.C. Andreozzi, A. Foerster, A. Rufle, L. Tornillo, and L. Terracciano. 2012. KRAS mutation testing in colorectal cancer: comparison of the results obtained using 3 different methods for the analysis of codons G12 and G13. Diagn. Mol. Pathol. 21:14-23.

13. McCourt, C.M., D.G. McArt, K. Mills, M.A. Catherwood, P. Maxwell, D.J. Waugh, P. Hamilton, J.M. O'Sullivan, and M. Salto-Tellez. 2013. Validation of next generation sequencing technologies in comparison to current diagnostic gold standards for BRAF, EGFR and KRAS mutational analysis. PLoS ONE 8:e69604.

14. Ogino, S., T. Kawasaki, M. Brahmandam, L. Yan, M. Cantor, C. Namgyal, M. Mino-Kenudson, G.Y. Lauwers, et al. 2005. Sensitive sequencing method for KRAS mutation detection by Pyrosequencing. J. Mol. Diagn. 7:413-421.

15. Whitehall, V., K. Tran, A. Umapathy, F. Grieu, C. Hewitt, T.J. Evans, T. Ismail, W.Q. Li, et al. 2009. A multicenter blinded study to evaluate KRAS mutation testing methodologies in the clinical setting. J. Mol. Diagn. 11:543-552.

16. Marchetti, A., M. Milella, L. Felicioni, F. Cappuzzo, L. Irtelli, G.M. Del, M. Sciarrotta, S. Malatesta, et al. 2009. Clinical implications of KRAS mutations in lung cancer patients treated with tyrosine kinase inhibitors: an important role for mutations in minor clones. Neoplasia 11:1084-1092.

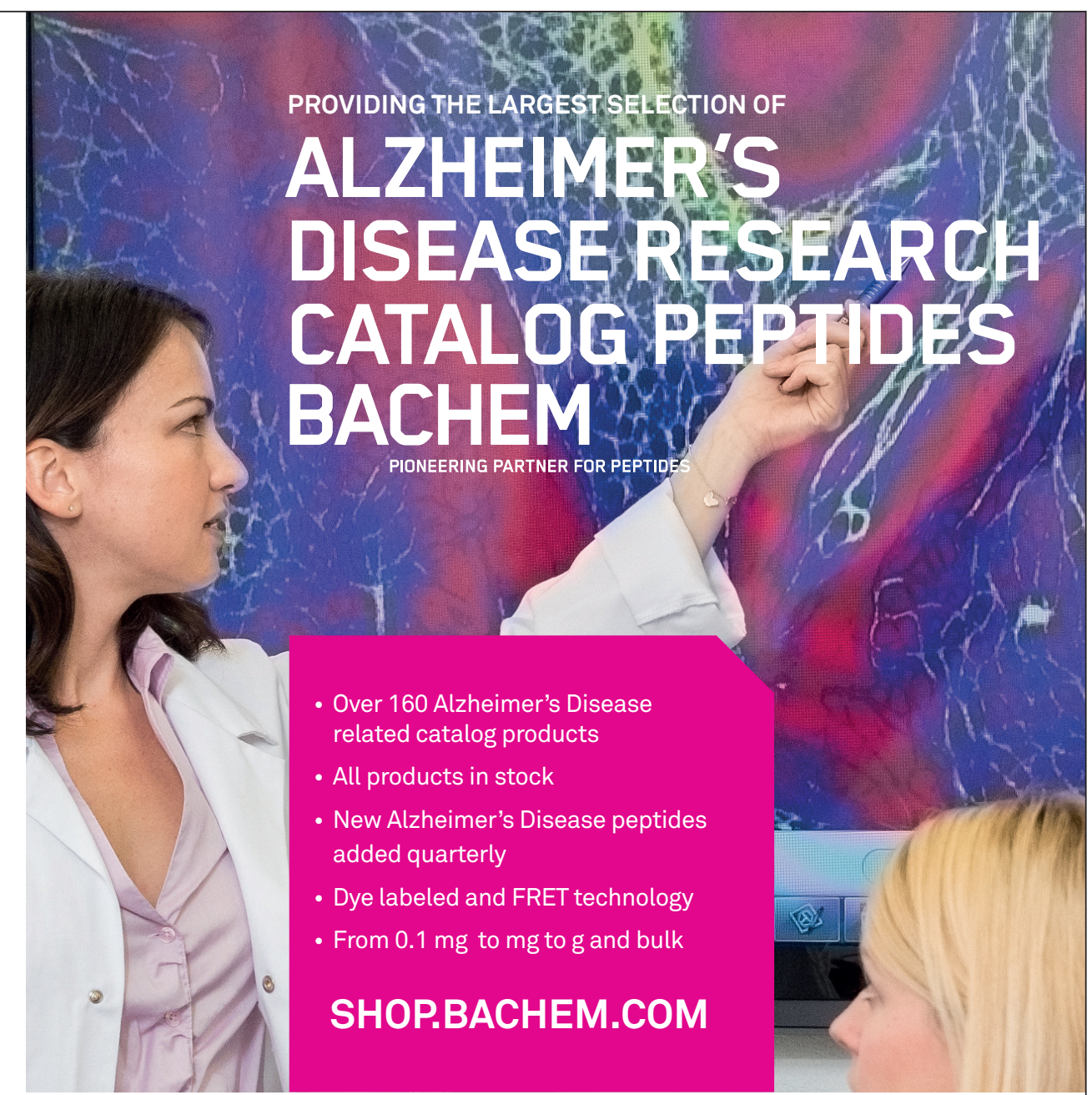

17. Turner, N.C. and J.S. Reis-Filho. 2012. Genetic heterogeneity and cancer drug resistance. Lancet Oncol. 13:e178-e185.

18. Palmirotta, R., G. Ludovici, M.L. De Marchis, B. Leone, V. Formica, G.M. Ettorre, F. Cavaliere, D. Della-Morte, et al. 2012. A comprehensive procedural approach to genotyping KRAS and $B R A F$ from paraffin embedded tissues for diagnostic purposes. In Vivo 26:537-547.

19. Vogelstein, B., N. Papadopoulos, V.E. Velculescu, S. Zhou, L.A. Diaz, Jr., and K.W. Kinzler. 2013. Cancer genome landscapes. Science 339:1546-1558.

20. Laghi, L., O. Orbetegli, P. Bianchi, A. Zerbi, V. Di Carlo, C. Boland, and A. Malesci. 2002. Common occurrence of multiple K-RAS mutations in pancreatic cancers with associated precursor lesions and in biliary cancers. Oncogene 21:43014306.

21. Benesova, L., M. Minarik, D. Jancarikova, B. Belsanova, and M. Pesek. 2010. Multiplicity of EGFR and KRAS mutations in non-small cell lung cancer (NSCLC) patients treated with tyrosine kinase inhibitors. Anticancer Res. 30:1667-1671.

22. Chen, J., Y. Ye, H. Sun, and G. Shi. 2013. Association between KRAS codon 13 mutations and clinical response to anti-EGFR treatment in patients with metastatic colorectal cancer: results from a meta-analysis. Cancer Chemother. Pharmacol. 71:265-272.

23. Altimari, A., D. de Biase, G. De Maglio, E. Gruppioni, E. Capizzi, A. Degiovanni, A. D’Errico, A. Pession, et al. 2013. 454 next generation-sequencing outperforms allelespecific PCR, Sanger sequencing, and pyrosequencing for routine KRAS mutation analysis of formalin-fixed, paraffin-embedded samples. Onco Targets Ther. 6:1057-1064.

24. Smits, A.J., J.A. Kummer, P.C. de Bruin, M. Bol, J.G. van den Tweel, K.A. Seldenrijk, S.M. Willems, G.J. Offerhaus, et al. 2014. The estimation of tumor cell percentage for molecular testing by pathologists is not accurate. Mod. Pathol. 27:168-174.

25. Sikkema-Raddatz, B., L.F. Johansson, E.N. de Boer, R. Almomani, L.G. Boven, M.P. van den Berg, K.Y. van Spaendonck-Zwarts, J.P. van Tintelen, et al. 2013. Targeted next-generation sequencing can replace Sanger sequencing in clinical diagnostics. Hum. Mutat. 34:1035-1042.

26. Furugaki, K., H. Yasuno, T. Iwai, Y. Moriya, N. Harada, and K. Fujimoto-Ouchi. 2014. Melting curve analysis for mutations of EGFR and KRAS. Anticancer Res. 34:613-621.

27. Han, S.W., H.P. Kim, J.Y. Shin, E.G. Jeong, W.C. Lee, K.H. Lee, J.K. Won, T.Y. Kim, et al. 2013. Targeted sequencing of cancer-related genes in colorectal cancer using next-generation sequencing. PLoS ONE 8:e64271.

28. Danzer, M., N. Niklas, S. Stabentheiner, K. Hofer, J. Proll, C. Stuckler, E. Raml, H. Polin, and C. Gabriel. 2013. Rapid, scalable and highly automated HLA genotyping using next-generation sequencing: a transition from research to diagnostics. BMC Genomics 14:221.

Received 13 June 2014; accepted 17 December 2014

Address correspondence to Markus Roucka, VelaLabs, Vienna, Austria. E-mail: m.roucka@ vela-labs.at

To purchase reprints of this article, contact: biotechniques@fosterprinting.com 